\title{
Gas6/Axl signaling pathway promotes proliferation, migration and invasion and inhibits apoptosis in A549 cells
}

\author{
DONG WANG ${ }^{1 *}$, LIXIN BI $^{1 *}$, JINGPING RAN $^{2}$, LEI ZHANG $^{3}$, NA XIAO $^{3}$ and XIAOLI LI ${ }^{3}$ \\ ${ }^{1}$ Department of Tuberculosis; ${ }^{2}$ Clinical Laboratory; ${ }^{3}$ Department of Medical Oncology, \\ Affiliated Hospital of Hebei University, Baoding, Hebei 071000, P.R. China
}

Received July 27, 2019; Accepted February 23, 2021

DOI: $10.3892 /$ etm.2021.10756

\begin{abstract}
Several studies have demonstrated that growth arrest-specific protein 6 (Gas6) and Axl are highly expressed in various tumor tissues, such as renal cell and esophageal carcinoma. However, the effect of the Gas6/Axl signaling pathway on lung adenocarcinoma is still unclear. The aim of the present study was to investigate the effect of the Gas6/Axl signaling pathway on lung adenocarcinoma cells and its mechanism of action, which may provide a novel target for the clinical treatment of lung adenocarcinoma. Human lung adenocarcinoma tissues were used to examine the activation of the Gas6/Axl signaling pathway. In addition, the human lung adenocarcinoma cell line A549 was employed to study the effects of the Gas6/Axl signaling pathway on the proliferation, migration, invasion and apoptosis of lung adenocarcinoma cells. Recombinant human Gas6 protein and inhibitor TP-0903 were used to activate and inhibit the Gas6/Axl signaling pathway, respectively. The results revealed that Gas6 and Axl expression level was increased in human lung adenocarcinoma tissues compared with adjacent healthy tissues. After inhibition of the Gas6/Axl signaling pathway with TP-0903, p21, p53, caspase 3, caspase 8 and caspase 9 exhibited higher expression level in A549 cells. The opposite effect was observed when the Gas6/Axl signaling pathway was activated. In addition, the migratory and invasive ability of A549 cells was determined via wound-healing and Transwell invasion assays. The results indicated that the migratory and invasive ability of A549 cells was significantly increased when the Gas6/Axl signaling pathway was activated and inhibition of Gas6/Axl signaling pathway caused the opposite results. Activity of Gas6/Axl signaling pathway was shown to be
\end{abstract}

Correspondence to: $\operatorname{Dr}$ Xiaoli Li, Department of Medical Oncology, Affiliated Hospital of Hebei University, 648 Dongfeng East Road, Baoding, Hebei 071000, P.R. China

E-mail: 57425183@qq.com

${ }^{*}$ Contributed equally

Key words: growth arrest-specific protein 6/Axl signaling pathway, proliferation, migration, invasion, apoptosis positively associated with cell proliferation by Cell Counting Kit 8 and clone formation assays. In conclusion, the Gas6/Axl signaling pathway was revealed to promote the proliferation, migration and invasion and inhibit the apoptosis of lung adenocarcinoma cells, which serve important roles in the progression of lung adenocarcinoma.

\section{Introduction}

At present, lung cancer is the leading cause of cancer-associated mortality, resulting in 30-40 deaths per 100,000 $(1,2)$. Histologically, lung cancer can be divided into small and non-small cell lung cancer (2). As one of the most common subtypes of non-small cell lung cancer, the incidence of lung adenocarcinoma accounts for $>40 \%$ of the total incidence of lung cancer (3). Moreover, in the past few decades the incidence of lung adenocarcinoma has demonstrated an upward trend, which has posed a great threat to human life and health (4). Therefore, the early diagnosis and treatment of lung adenocarcinoma is important for the prevention and treatment of lung cancer. However, early diagnosis of lung adenocarcinoma is difficult, as the early symptoms of lung adenocarcinoma are insignificant, and no specific markers exist (5). When cancer occurs, patients are usually in a locally advanced stage, but the patients are usually diagnosed in the terminal stage of cancer. Therefore, patients miss the optimal opportunity for surgical treatment (6). Although traditional anti-tumor treatments, such as radiotherapy and chemotherapy, can exhibit certain curative effects, they cannot markedly change the mortality rate of lung adenocarcinoma due to their toxic effects on normal cells and multi-drug resistance (7). Previously, tumor-targeted therapeutic drugs, such as gefitinib and cetuximab, have achieved certain effects, but their application range is narrow, and various side effects have also occurred and resistance has emerged (8). Therefore, the search for novel and effective approaches for early diagnosis and treatment still constitute an important element for the prevention and treatment of lung adenocarcinoma.

Axl is a member of the receptor tyrosine kinase family. It binds to its ligand growth arrest-specific protein 6 (Gas6) and activates its own tyrosine kinase activity (9). It activates downstream signal transduction pathways and serves an important role in cell adhesion, proliferation, migration and inhibition of apoptosis (9). Gas6 and Axl have been indicated 
to be highly expressed in various tumor tissues, and activation of the Gas6/Axl signaling pathway has been revealed to serve an important role in tumor cell proliferation and inhibition of apoptosis, tumor angiogenesis, tumor invasion and metastasis $(10,11)$. Sawabu et al (12) demonstrated that the expression of Gas6 and Axl mRNA and protein was higher in gastric cancer tissues compared with adjacent healthy tissues. In a study on hepatoma cells lines, Lee et al (13) indicated that Gas6 induced Axl phosphorylation to activate the MAPK/ERK signaling pathway and upregulate the downstream factor Slug. Slug belongs to the Snail transcriptional repressor superfamily, and its upregulation can enhance cell motility, thereby enhancing hepatoma cell invasive ability (13). However, in lung adenocarcinoma, the role of the Gas6/Axl signaling pathway has rarely been studied. Therefore, the current study used human lung adenocarcinoma tissues and the human lung adenocarcinoma cell line A549 to study the effects of the Gas6/Axl signaling pathway on lung adenocarcinoma. The present study demonstrated that TP-0903, which is an inhibitor of the Gas6/Axl signaling pathway, may be a targeted drug for the Gas6/Axl signaling pathway for the treatment of lung adenocarcinoma as a better alternative to other drugs, such as gefitinib and cetuximab.

\section{Materials and methods}

Patient tissue samples. All lung adenocarcinoma tissues were obtained from patients undergoing lung adenocarcinoma resection. The present study was approved by the Ethics Committee of the Affiliated Hospital of Hebei University (Baoding, China) and written informed consent was provided by all patients. All patients were diagnosed with lung adenocarcinoma by imaging and based on pathological findings. A total of 22 lung adenocarcinoma tissues (age, 55-65 years; 17 males and 5 females) and adjacent healthy tissues (distance, $1 \mathrm{~cm}$ ) were obtained from January 2017 to September 2019. Patients with first-onset disease were included; patients who had undergone radiotherapy or chemotherapy were excluded. During the operation, the lung adenocarcinoma and adjacent healthy tissues were immediately removed and stored in liquid nitrogen for subsequent experiments.

Cell culture and drug treatment. The lung adenocarcinoma cell line A549 was purchased from American Type Culture Collection. Cells were cultured in RPMI-1640 medium (Thermo Fisher Scientific, Inc.) containing $1 \times 10^{5} \mu \mathrm{mol} / 1$ penicillin and streptomycin and 10\% FBS (Thermo Fisher Scientific, Inc.). Cells were cultured in an incubator at $37^{\circ} \mathrm{C}$ with 5\% $\mathrm{CO}_{2}$. Then, $0.1 \mu \mathrm{M}$ Axl inhibitor (cat. no. TP-0903; Selleck Chemicals) (14), $1 \mu \mathrm{g} / \mathrm{ml}$ recombinant human Gas6 (ACROBiosystems, Inc.) (15), Gas6 + siRNA-Axl or an equal volume of PBS was used to treat cells. Control cells were treated with sterile phosphate buffer or negative control siRNA.

Small interfering (si)RNA transfection. A549 cells were transfected with siRNA-Axl or siRNA-negative control (Thermo Fisher Scientific, Inc.). A total of $95 \%$ of the cells were viable $12 \mathrm{~h}$ after transfection. The cells were incubated for another day before subsequent experiments. Axl expression level was decreased after transfection with siRNA-Axl. The transfection efficiency was examined via reverse transcription-quantitative PCR (RT-qPCR). The siRNA-Axl sequence was as follows: siRNA-Axl-sense, 5'-UAUCACAGGUGCCAGAGGA-3'; siRNA-Axl-antisense, 5'-UCCUCUGGCACCUGUGAUA-3'. Negative control siRNA sequence was 5'-GCAAGCTGACCC TGAAGTT-3'.

The cells were transfected after cell growth density had reached $30 \%$. A total of 50 pmol siRNA were mixed with serum-free RPMI-1640 medium in $25 \mu \mathrm{l}$ final volume, and $1 \mu 1$ Entranster-R4000 (Engreen Biosystem, Ltd.) was mixed with $24 \mu \mathrm{l}$ serum-free RPMI-1640 medium and incubated at room temperature for $15 \mathrm{~min}$. The RNA and Entranster-R4000 solutions were subsequently mixed and further incubated at room temperature for $15 \mathrm{~min}$. The $50 \mu \mathrm{l}$ transfection solution was mixed with $0.45 \mathrm{ml}$ complete RPMI-1640 medium and added into the cells. A total of $6 \mathrm{~h}$ after transfection at $37^{\circ} \mathrm{C}$, the cell medium was replaced with fresh complete RPMI-1640 medium and the cells were cultured.

Western blot analysis. A549 cells were seeded in six-well plates at $1 \times 10^{5}$ cells/well. After the cells had reached the appropriate confluency at 2 days, total protein was extracted using RIPA lysis buffer (Beyotime Institute of Biotechnology), and protein concentration was measured using the BCA method (Thermo Fisher Scientific, Inc.). SDS-PAGE was subsequently performed with $10 \%$ gels and $30 \mu \mathrm{g}$ protein loaded per lane, followed by transfer of the proteins to a PVDF membrane. After blocking with 5\% BSA (Beyotime Institute of Biotechnology) for $2 \mathrm{~h}$ at room temperature, the PVDF membrane was incubated with the following primary antibodies: Gas6 (rabbit; 1:3,000; Abcam; cat. no. ab264098), Axl, (rabbit; 1:3,000; Abcam; cat. no. ab259831), p21 (rabbit; 1:3,000; Abcam; cat. no. ab109520), p53 (rabbit; 1:3,000; Abcam; cat. no. ab32389), caspase-3 (rabbit; 1:1,000; Cell Signaling Technology, Inc.; cat. no. 9662S), caspase-9 (rabbit; 1:1,000; Abcam; cat. no. ab32539) and $\beta$-actin (1:10,000; ProteinTech Group, Inc.; cat. no. 66009-1-Ig) overnight at $4^{\circ} \mathrm{C}$. Following three washes with TBS-0.1\% Tween 20 (TBST), the membrane was incubated in the secondary antibody solution (horseradish peroxidase-conjugated goat anti-rabbit IgG H\&L; 1:2,000; Abcam; cat. no. ab205718) on a shaker for $1 \mathrm{~h}$ at room temperature. After washing the membrane three times using TBST, the protein bands were visualized and detected using an electrochemiluminescence system (Sigma-Aldrich; Merck $\mathrm{KGaA})$. $\beta$-actin was used as the internal control.

$R T$-qPCR. A549 cells were seeded into 6-well plates at $1 \times 10^{5}$ cells/well, and the cells were treated as aforementioned. TRIzol ${ }^{\circledR}$ reagent (Thermo Fisher Scientific, Inc.) was used to extract total RNA from the cells. A spectrophotometer (UV-670; Shanghai Mapada Instruments Co., Ltd.) was used to measure the concentration of the extracted RNA, and the RNA concentration was diluted to $200-500 \mathrm{ng} / \mu \mathrm{l}$ with RNase free water. Following measurement of the RNA concentration, RNA was reverse transcribed to cDNA using the PrimeScript $^{\mathrm{TM}}$ RT Master Mix (Thermo Fisher Scientific, Inc.). Temperature protocol was $15 \mathrm{~min}$ at $37^{\circ} \mathrm{C}, 5 \mathrm{sec}$ at $85^{\circ} \mathrm{C}$ and $30 \mathrm{~min}$ at $4^{\circ} \mathrm{C}$. Subsequently, qPCR was performed to quantify Gas6, Axl, p21, p53, caspase-3 and caspase-9 expression level 
Table I. Primers used for reverse transcription-quantitative PCR.

\begin{tabular}{llll}
\hline Gene name & Primer type & \multicolumn{1}{c}{ Sequence $\left(5^{\prime}-3^{\prime}\right)$} & Length (base no.) \\
\hline Axl & Forward & TCAAGGTGGCTGTGAAGACGA & 21 \\
& Reverse & CGTTCAGAACCCTGGAAACAGAC & 23 \\
Gas6 & Forward & GCCTTCTACAGCCTGGACTAC & 21 \\
& Reverse & TCTTGAGTTTCTTCGTGGAGTG & 22 \\
p21 & Forward & AGTATGCCGTCGTCTGTTCG & 20 \\
p53 & Feverse & GACTGCAAGACAGCGACAAG & 21 \\
& Forward & GGTTCCTGCCCCAGGATGTTG & 20 \\
Caspase-3 & Reverse & GGAACATCTCGAAGCGCTCA & 20 \\
& Forward & CAGAATCATAAGCCCCTGGA & 19 \\
Gaspase-9 & Reverse & TCTGCGAGTCAGGCATTTG & 18 \\
& Forward & TTCTTGAGCAACACCCTC & 22 \\
& Reverse & CGCATACACTGTCTACCT & 19 \\
\hline
\end{tabular}

Gas6, growth arrest-specific protein 6 .

using TaqMan Fast Advanced Master Mix (Thermo Fisher Scientific, Inc.) according to the manufacturer's instructions, and GAPDH was used for normalization. The primers used for RT-qPCR are presented in Table I. Relative mRNA expression levels were calculated using the $2^{-\Delta \Delta \mathrm{Cq}}$ method (16).

Immunocytofluorescence (IF) staining. Cell slides were placed into a 24-well plate and A549 cells were seeded on the slides at $5 \times 10^{4}$ cells/well. After cell confluence reached $50 \%$, IF staining was performed. After being washed with PBS, the cells were fixed with $4 \%$ paraformaldehyde for $20 \mathrm{~min}$ at room temperature and incubated in $1 \%$ Triton for $15 \mathrm{~min}$ at room temperature, followed by blocking non-specific antigens with $10 \%$ goat serum (Beyotime Institute of Biotechnology) for $1 \mathrm{~h}$ at room temperature. The cells were then incubated overnight at $4^{\circ} \mathrm{C}$ with caspase 8 (rabbit; 1:500; Abcam; cat. no. ab25901). The following day, after washing the cells with PBS, a fluorescent secondary antibody [goat anti-rabbit IgG H\&L (Alexa Fluor ${ }^{\circledR}$ 488); 1:500; Abcam; cat. no. ab150077] was added to the cells for $2 \mathrm{~h}$ at room temperature. Finally, DAPI was added and incubated for $10 \mathrm{~min}$ at room temperature. The cells were visualized using a fluorescence microscope (magnification x200; Carl Zeiss AG).

Cell Counting Kit-8 (CCK-8) assay. A total of 5,000 A549 cells/well were added to a 96 -well plate and placed in a $37^{\circ} \mathrm{C}$ cell culture incubator. After the cells became adherent, the cells were treated as aforementioned. Following treatment for $24 \mathrm{~h}, 10 \mu \mathrm{l} \mathrm{CCK}-8$ reagent (Dojindo Molecular Technologies, Inc.) was added to each well. The 96-well plate was then placed in the cell culture incubator for another $2 \mathrm{~h}$. Finally, a microplate reader was used to measure the absorbance of each well at $450 \mathrm{~nm}$.

Flow cytometry. A total of $1 \times 10^{5}$ A549 cells/well were seeded in a six-well plate. After cell confluence reached 50\%, flow cytometry was performed. The medium was discarded following treatment as aforementioned for $24 \mathrm{~h}$. Following washing of the cells with PBS, trypsin without EDTA was used to digest the cells for $2 \mathrm{~min}$ at $37^{\circ} \mathrm{C}$. Following collection by centrifugation $\left(1,000 \mathrm{x} \mathrm{g}, 5 \mathrm{~min}, 20^{\circ} \mathrm{C}\right)$, the cells were washed with cold PBS and were suspended in $400 \mu 11 \mathrm{X}$ Annexin V binding solution at $\sim 1 \times 10^{6}$ cells $/ \mathrm{ml}$. Subsequently, $5 \mu \mathrm{l}$ Annexin V-FITC (Beyotime Institute of Biotechnology) was added to each tube of cells, which was gently mixed and incubated in the dark for $15 \mathrm{~min}$ at room temperature, followed by addition of $10 \mu \mathrm{l} \mathrm{PI}$ in each tube and incubation for $5 \mathrm{~min}$ at room temperature. Finally, flow cytometry (Thermo Fisher Scientific, Inc.; cat. no. A28997) was used to detect apoptosis with Attune NxT software version 3.1.2 (Thermo Fisher Scientific, Inc.).

Wound-healing assay. A549 cells were seeded into a six-well plate at $1 \times 10^{5}$ cells/well. When cell confluence reached $100 \%$, a $10-\mu 1$ pipette tip was used to create wounds vertically in the wells. Following removal of the floating cells using PBS, the cells were cultured in serum-free RPMI-1640 medium containing aforementioned treatments. Photos (magnification x100) of the cells at the same position of the wound were captured under a light microscope at 0, 6, 12 and $24 \mathrm{~h}$ after wound creation to compare the healing speed of each group.

Transwell invasion assay. A total of $50 \mu 1$ Matrigel were added into the Transwell chambers $(8 \mu \mathrm{m})$ and incubated at $37^{\circ} \mathrm{C}$ for 30 min to allow Matrigel precoating. The treated cells were digested using trypsin for $2 \mathrm{~min}$ at room temperature, and the cell suspensions with $1 \times 10^{5}$ cells diluted in RPMI-1640 medium were added to the Transwell chambers. The serum-free medium was placed in the upper chamber and medium with $30 \%$ FBS was placed in the lower chamber. After incubation for $24 \mathrm{~h}$ at $37^{\circ} \mathrm{C}$ with $5 \% \mathrm{CO}_{2}$, the cells in the upper layer of the chamber filter were removed with a cotton swab. Subsequently, 
A
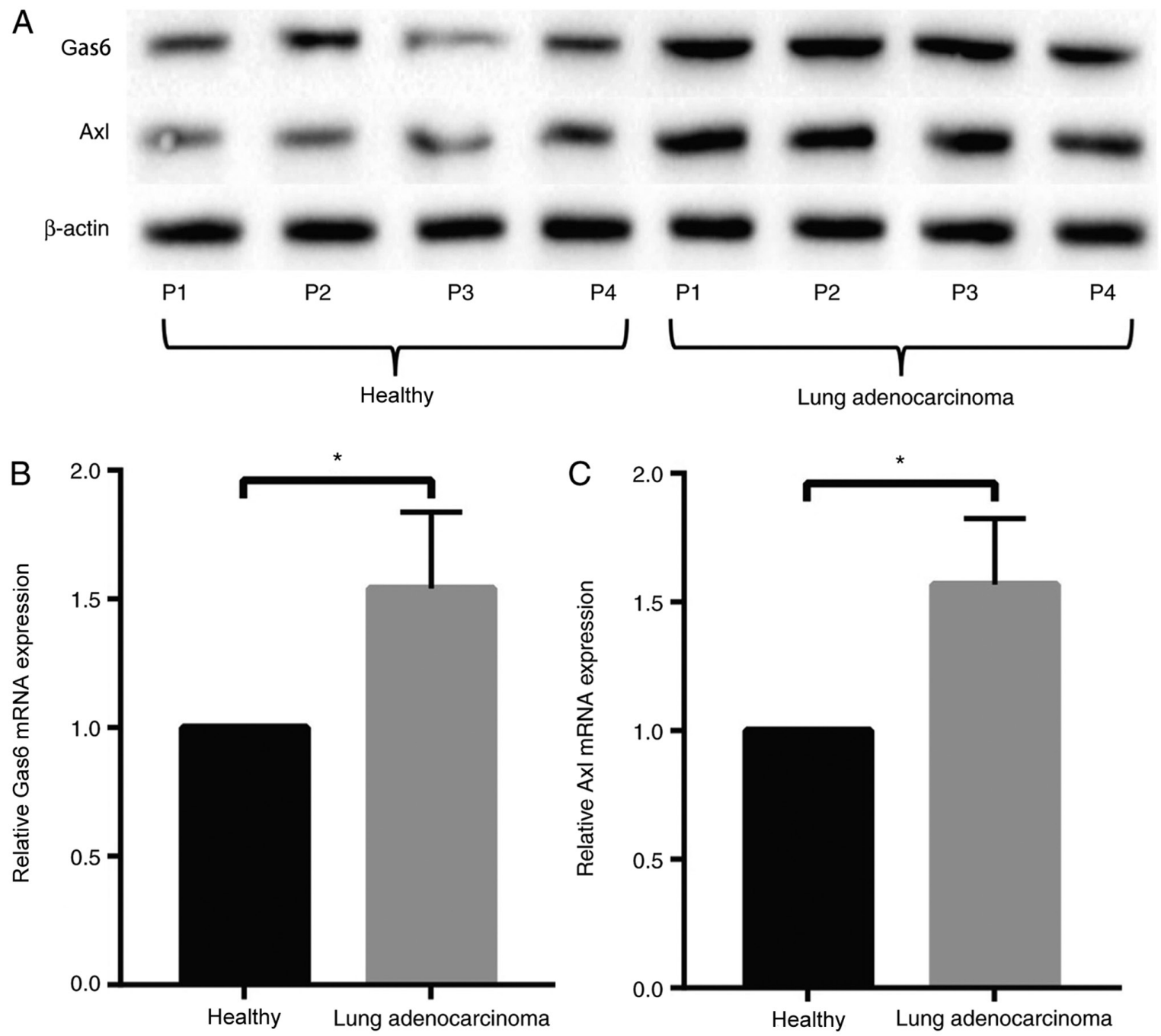

Figure 1. Lung adenocarcinoma tissues exhibit higher expression level of Gas6 and Axl compared with healthy adjacent tissues. The expression level of Gas6 and Axl in lung adenocarcinoma tissues and healthy adjacent tissues was determined by (A) western blotting and (B and C) RT-qPCR. A total of 4 lung adenocarcinoma and adjacent healthy tissues from 4 patients (P1, P2, P3 and P4) were randomly selected for western blotting, while all lung adenocarcinoma and adjacent healthy tissues were used for RT-qPCR. "P<0.05. RT-qPCR, reverse transcription-quantitative PCR; Gas6, growth arrest-specific protein 6.

$0.1 \%$ crystal violet was used to stain the invaded cells for $15 \mathrm{~min}$ at room temperature. The cells attached to the bottom layer of the chamber filter were observed by a light microscope (magnification $\mathrm{x} 400$ ), the number of cells was counted in four fields of view per sample, and the effects of treatment as aforementioned on the invasive ability of A549 cells were examined.

Colony formation assay. A549 cells $\left(5 \times 10^{3}\right.$ cells/well) in 24-well culture plates for each group were cultured for 7 days until the colonies (diameter, 0.3-1.0 mm) were visible. $0.1 \%$ crystal violet staining was performed for $30 \mathrm{~min}$ at room temperature, and the number of colonies was counted using ImageJ 1.52 software (National Institutes of Health).

Statistical analysis. STATA v12.0 software (StataCorp LP) was used for statistical analysis. Measurement data are presented as the mean \pm SD. A paired Student's t-test was used to compare data between two groups. For comparisons among $>2$ groups, one-way ANOVA followed by Bonferroni's post hoc test was used. All experiments were repeated three times.
$\mathrm{P}<0.05$ was considered to indicate a statistically significant difference.

\section{Results}

High expression of Gas6 and Axl in lung adenocarcinoma tissues. Lung adenocarcinoma tissues removed during surgery were compared with adjacent healthy tissues, and the expression level of Gas6 and Axl was detected by western blotting (Fig. 1A) and RT-qPCR (Fig. 1B and C). The results revealed that the expression level of Gas6 and Axl in lung adenocarcinoma tissues was notably higher compared with that of the adjacent healthy tissues.

Gas6/Axl signaling pathway promotes proliferation in A549 cells. The effect of Gas6/Axl signaling pathway on lung adenocarcinoma cells was demonstrated using two different ways of inhibiting the Gas6/Axl signaling pathway. TP-0903 and siRNA-Axl were used as inhibitors of the Gas6/Axl signaling pathway. RT-qPCR revealed that transfection with siRNA-Axl efficiently reduced the expression level of the Axl 
A

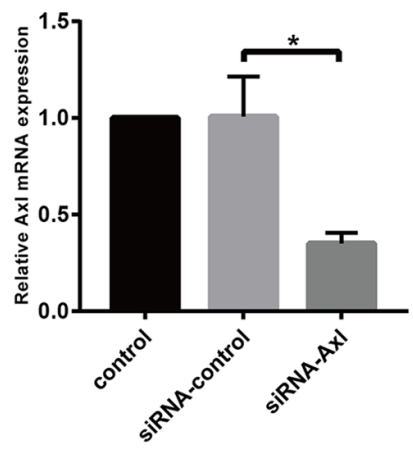

E

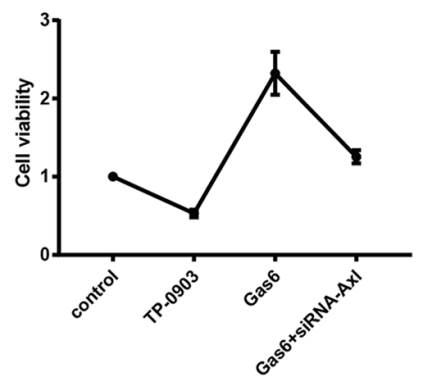

B

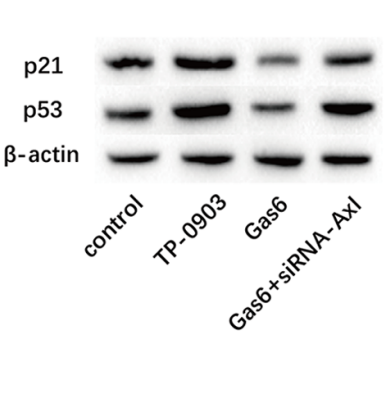

F

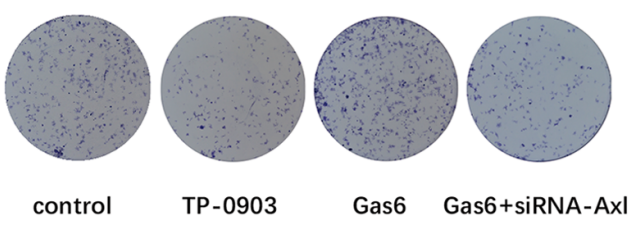

C

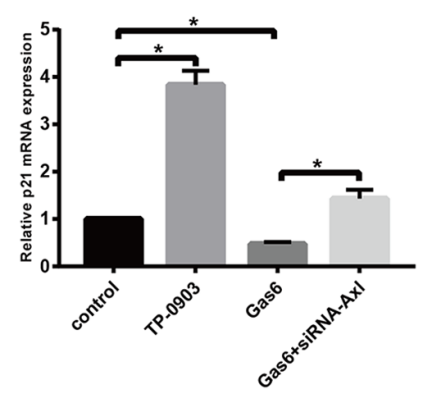

D

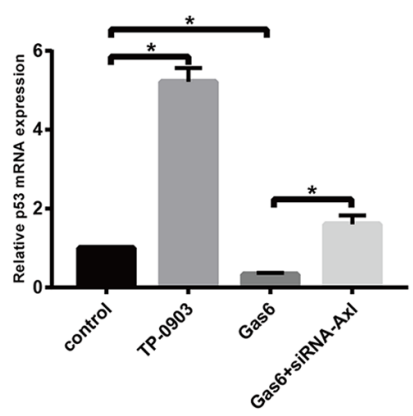

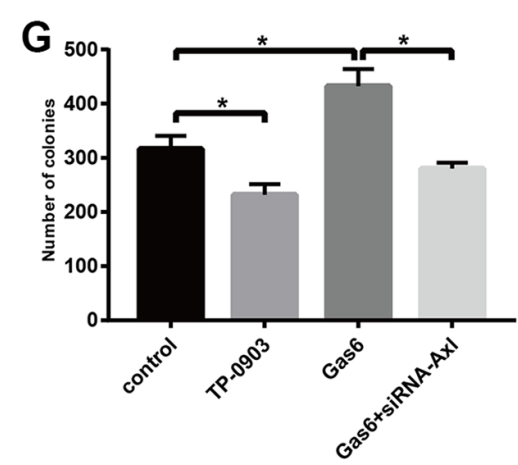

Figure 2. Gas6/Axl signaling pathway promotes proliferation in A549 cells. (A) The efficiency of siRNA-Axl transfection was determined via RT-qPCR. (B) The expression level of p21 and p53 in A549 cells was determined by western blotting and (C and D) RT-qPCR. The proliferative ability of the indicated groups was determined using (E) Cell Counting Kit-8 assay and (F and G) colony-formation assay. ${ }^{*} \mathrm{P}<0.05$. RT-qPCR, reverse transcription-quantitative PCR; Gas6, growth arrest-specific protein 6; siRNA, small interfering RNA.

mRNA compared with the siRNA-control (Fig. 1A). The results of western blotting (Fig. 2B) and RT-qPCR (Fig. 2C and D) demonstrated that TP-0903 increased, while recombinant human Gas6 decreased the expression level of p21 and p53 compared with control cells. Furthermore, the expression level of p21 and p53 in the Gas6 + siRNA-Axl group higher compared with that of the Gas 6 group. In addition, CCK-8 assay (Fig. 2E) indicated that the inhibitor TP-0903 decreased and recombinant human Gas6 increased cell viability when compared with the control group. Furthermore, cell viability was decreased in the Gas6 + siRNA-Axl group compared with the Gas6 group. The results of the colony formation assay were similar to those of the CCK8 assay (Fig. 2F and G). The results revealed that the proliferation of A549 cells also decreased or increased by inhibiting or activating the Gas6/Ax1 signaling pathway, respectively.

Gas6/Axl signaling pathway promotes the migration and invasion of A549 cells. The effect of the Gas6/Axl signaling pathway on the migratory and invasive ability of A549 cells was examined using wound-healing and Transwell invasion assays. The results of the wound-healing assay (Fig. 3A and C) demonstrated that the cell scratch healing rate was lower in the TP-0903 group and higher in the Gas6 group when compared with the control group, and the cell scratch rate of the Gas6 + siRNA-Axl group was lower when compared with the Gas6 group. The Transwell invasion assay (Fig. 3B and D) revealed that when compared with the control group, the cell invasive ability of the TP-0903 group was decreased and that of the Gas6 group was increased. Furthermore, the cell invasive ability of the Gas6 + siRNA-Axl group was reduced compared with that of the Gas 6 group. Therefore, the results indicated that the migratory and invasive ability of A549 cells decreased or increased by inhibiting or activating the Gas6/Axl signaling pathway, respectively.

Gas6/Axl signaling pathway inhibits apoptosis in A549 cells. The apoptosis of A549 cells was examined by flow cytometry (Fig. 4A and B). The results revealed that the apoptotic level of the TP-0903 group was higher and that of the Gas6 group was lower compared with that of the control group, and the level of apoptosis in the Gas6 + siRNA-Axl group was higher compared with that of the Gas6 group. Western blot analysis (Fig. 4C) and RT-qPCR (Fig. 4D and E) demonstrated that the expression level of caspase- 3 and caspase- 9 was increased in the TP-0903 group and decreased in the Gas6 group compared with the control, but the expression level of caspase- 3 and caspase-9 in the Gas6 + siRNA-Axl group was higher than that of the Gas 6 group. The results of cell immunofluorescence (Fig. 4F) indicated that TP-0939 increased and Gas6 reduced the expression of caspase- 8 , while siRNA-Axl could attenuate the effect of Gas6. These results indicated that when the Gas6/Ax1 signaling pathway was inhibited or activated, the level of apoptosis of A549 cells increased or decreased, respectively.

\section{Discussion}

Lung adenocarcinoma is the most common subtype of lung cancer, accounting for $65 \%$ of non-small cell lung cancer cases 
A

$\mathrm{Oh}$

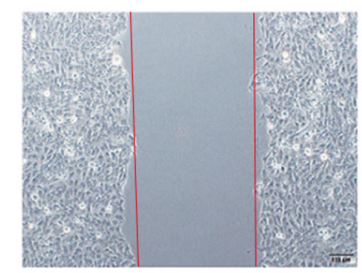

$6 \mathrm{~h}$

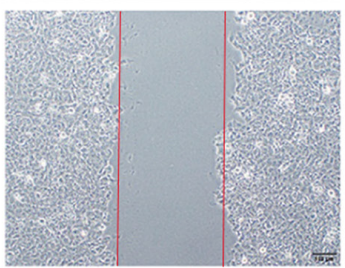

$12 \mathrm{~h}$

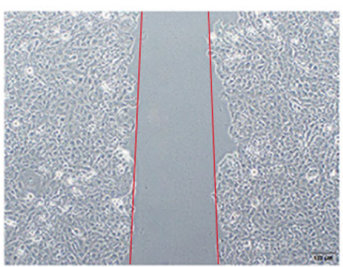

$24 \mathrm{~h}$

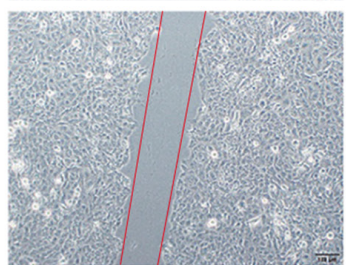

Control
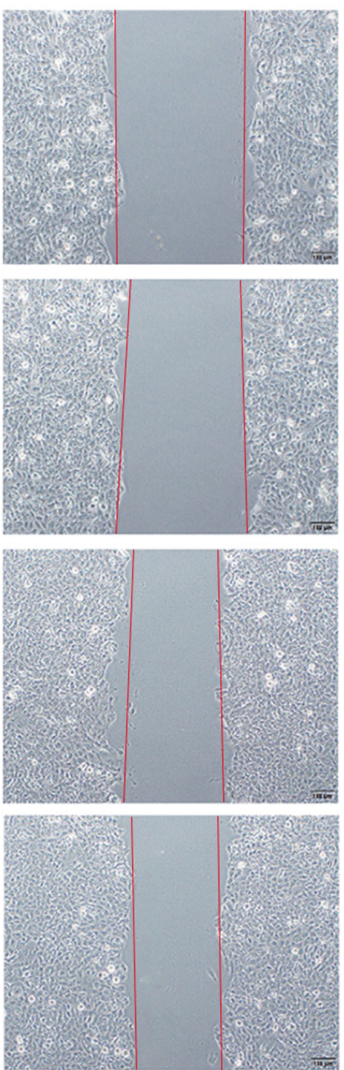

TP-0903
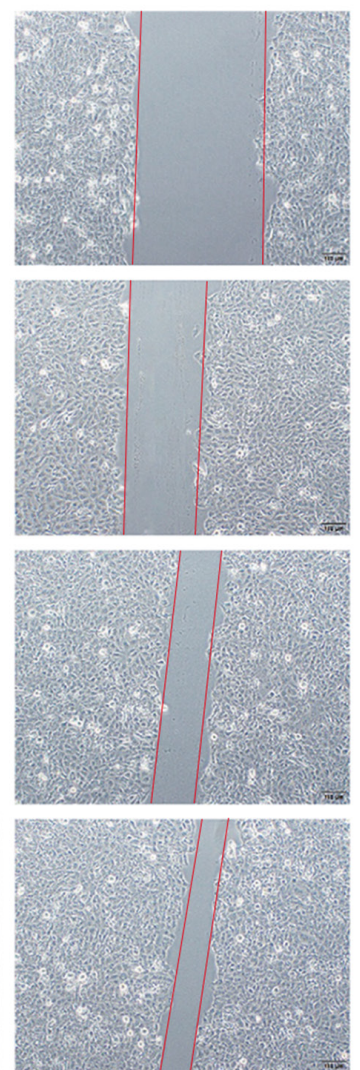

Gas6
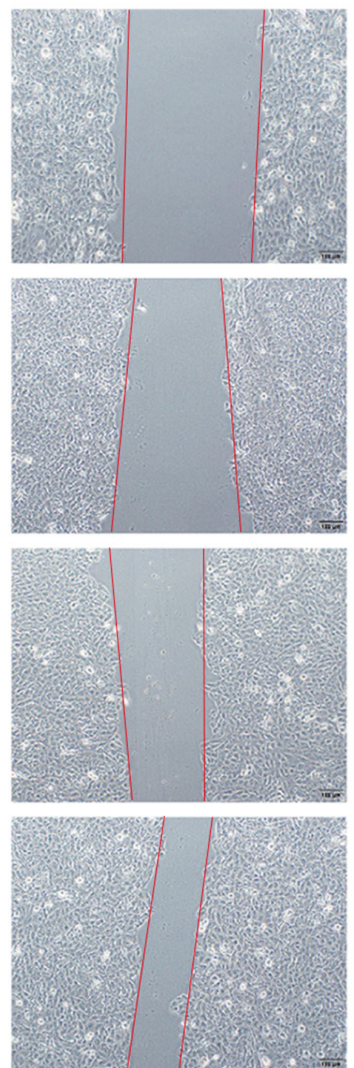

Gas6+siRNA-Ax

B

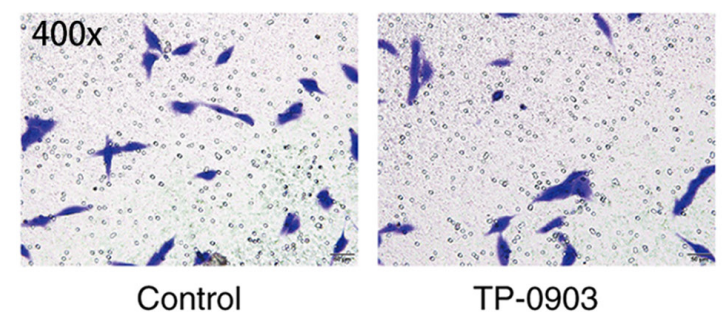

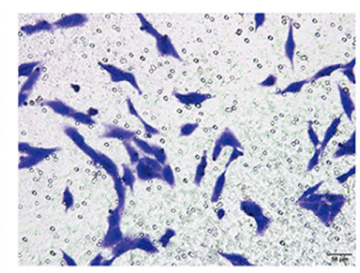

Gas6

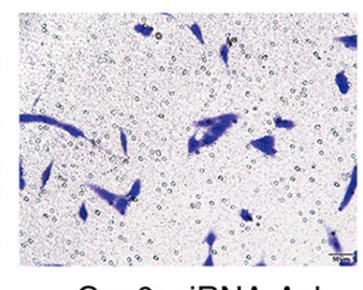

Gas6+siRNA-Axl
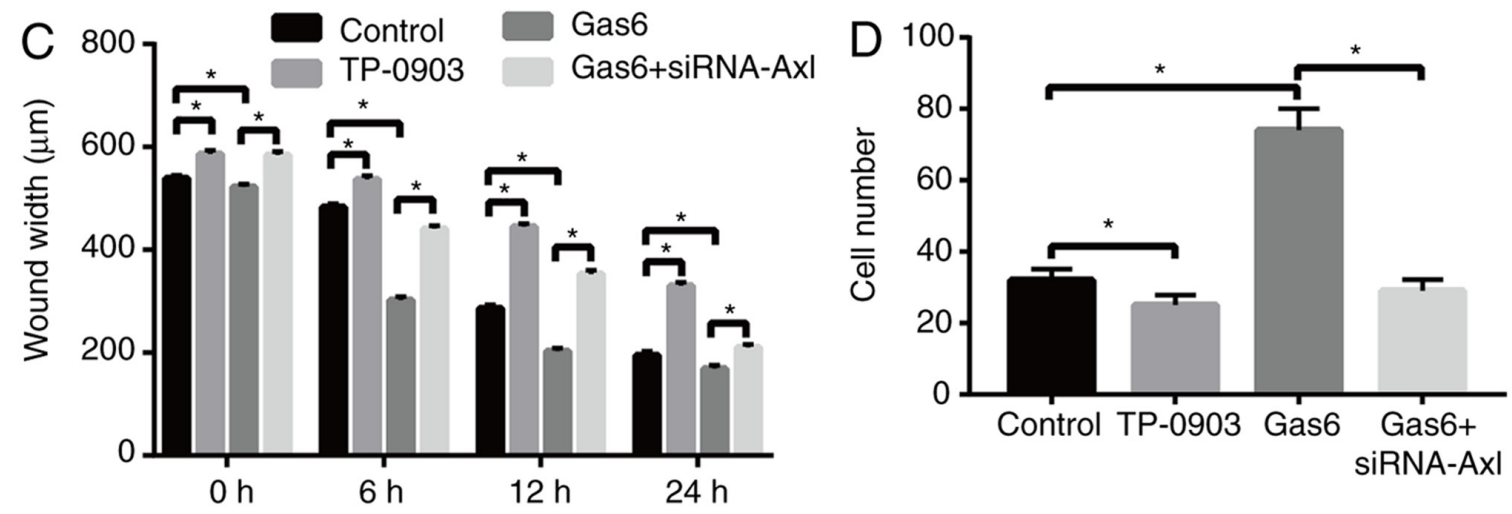

Figure 3. Gas6/Axl signaling pathway promotes the migration and invasion of A549 cells. The migratory and invasive ability of the indicated groups was determined via (A and C) wound-healing assay and (B and D) Transwell invasion assay, respectively. ${ }^{*} \mathrm{P}<0.05$. Scale bars, $50 \mu \mathrm{m}$. Gas6, growth arrest-specific protein 6; siRNA, small interfering RNA.

and $>40 \%$ of all lung cancers (17). Therefore, understanding the carcinogenic signaling mechanisms of lung adenocarcinoma is necessary to discover novel therapeutic targets for the treatment of this disease. In the present study, the Gas6/Axl signaling pathway was demonstrated to increase the proliferation, invasion and migration of A549 cells, and inhibit the apoptosis of A549 cells. In addition, high expression level of Gas6 and Axl was also observed in the lung tissues of patients with lung adenocarcinoma. These data confirmed the pro-tumorigenic role of the Gas6/Axl signaling pathway. These results are 
A
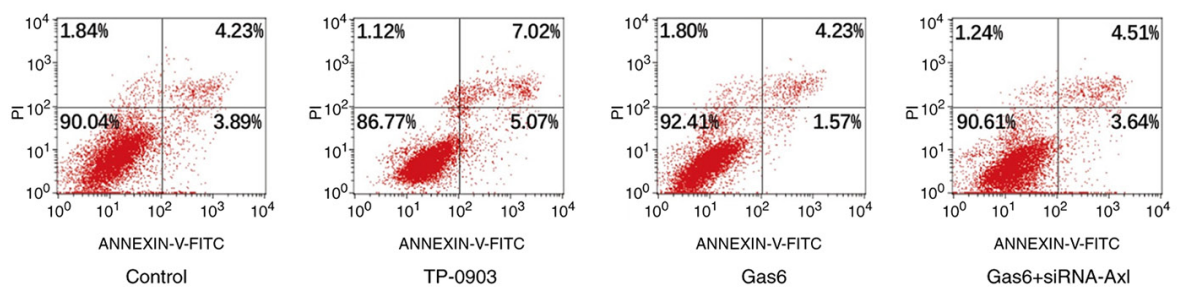
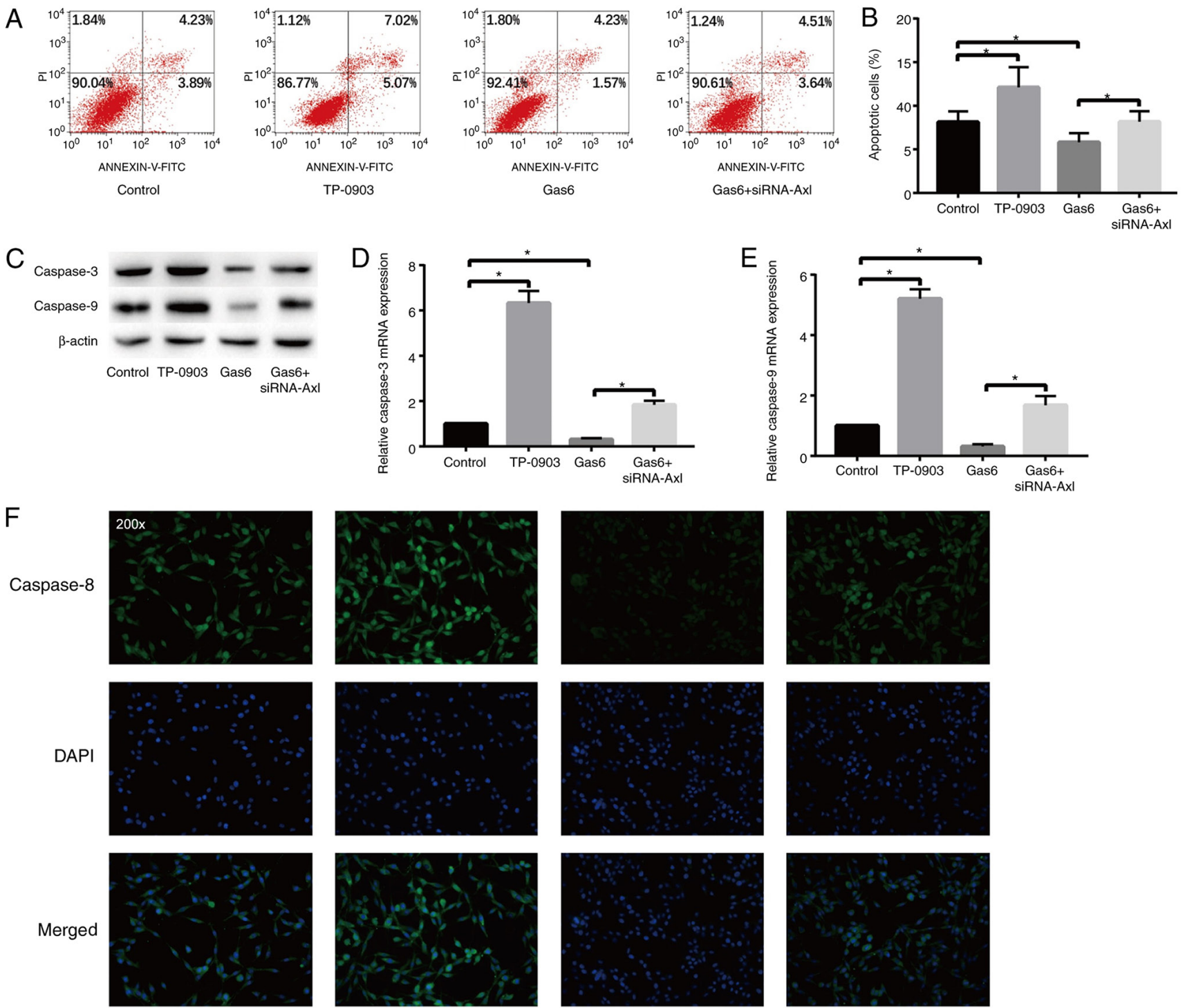

Control

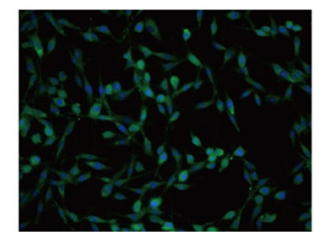

TP-0903

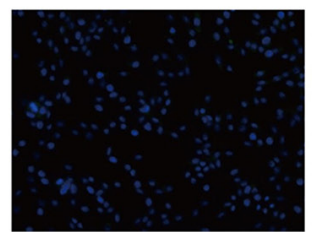

Gas6

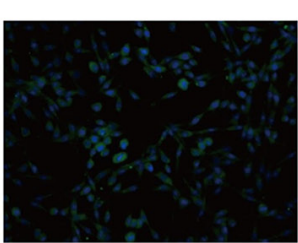

Gas6+siRNA-Ax

Figure 4. Gas6/Axl signaling pathway inhibits apoptosis in A549 cells. (A and B) The level of apoptosis in the indicated groups was determined by flow cytometry. The protein and mRNA expression level of caspase-3 and caspase-9 in the indicated groups was determined by (C) western blotting and (D and E) reverse transcription-quantitative PCR. (F) The expression level of caspase-8 in the indicated groups was determined by cell immunofluorescence. ${ }^{*} \mathrm{P}<0.05$. Gas6, growth arrest-specific protein 6; siRNA, small interfering RNA.

similar to those by Kariolis et al (18), who demonstrated that inhibiting the Gas6/Axl signaling pathway potentiated the anti-tumor effect of chemotherapy. Wang et al (19) also revealed that the Gas6/Axl axis can promote breast cancer resistance and metastasis via the AKT/GSK-3 $\beta / \beta$-catenin signaling pathway. These results indicated that the Gas6/Axl signaling pathway promoted malignant tumorigenesis.

As a common neoplastic disease, lung adenocarcinoma has a great impact on human health (20). When patients visit their doctor are often in the late stages of the disease; therefore, it is particularly important to discover targets for the treatment of lung adenocarcinoma (21). The present study revealed that the Gas6/Axl signaling pathway exhibited an important effect on lung adenocarcinoma. When the Gas6/Axl signaling pathway was inhibited, the proliferation and invasive ability of lung adenocarcinoma cells were significantly decreased and the apoptotic level was increased. These results are similar to those of previous studies. For instance, in an in vitro study in osteosarcoma cell lines, Han et al (22) demonstrated that the expression of phosphorylated (p)-AKT in the osteosarcoma MG63 and U2OS cell lines was associated in a concentration-dependent manner with exogenous Gas6 and p-AKT expression, and that p-Axl was positively associated with p-AKT. It was also demonstrated that exogenous Gas6 attenuated the apoptosis rate of these osteosarcoma cell lines by activating Axl, and it was hypothesized that the Gas6/Axl signaling pathway may promote osteosarcoma by activating the downstream PI3K/AKT signaling pathway (22). In the A549 lung adenocarcinoma cell line of the present study, the inhibitory effect of the Gas6/Axl pathway on tumor cell apoptosis was also demonstrated. However, it is unclear whether the Gas6/Axl signaling pathway exerts an anti-apoptotic effect by activating the PI3K/AKT signaling pathway in lung adenocarcinoma. Therefore, the mechanism of the anti-apoptotic effect of the Gas6/Axl signaling pathway on tumor cells will be further explored in future studies. Rankin et al (23) revealed 
that downregulation of Axl expression in ovarian cancer cells inhibited MMP-2 gene transcription by decreasing the activity of the MMP-2 promoter, whereas decreased MMP-2 expression resulted in decreased invasion, metastasis and tumorigenic ability of the ovarian cancer cells. The study also demonstrated that the expression level of p-AKT was decreased when Axl expression was downregulated in ovarian cancer cells, while the expression level of p-ERK1/2 was not affected (23). Therefore, it was speculated that the Gas6/Axl axis promoted the activation of the PI3K/AKT pathway, which in turn enhanced the expression and activity of MMP-2. As a result, MMP-2 promoted the invasion and metastasis of tumor cells. Sainaghi et al (24) demonstrated that when exogenous Gas6 was added into the culture medium of the DU145 prostate cancer cell line during serum starvation, the uptake of ${ }^{[3 \mathrm{H}]}$-thymidine by tumor cells increased, and the expression level of p-AKT and p-p38 MAPK also increased. This suggested that Gas6 may enhance the mitotic ability of tumor cells by activating the PI3K/AKT and p38 MAPK signaling pathways. Therefore, existing studies have indicated that the Gas6/Axl signaling pathway can promote tumor cell migration and invasion, while increasing tumor cell proliferation in various types of tumors.

A previous study has also demonstrated that Gas6 and Axl were highly expressed in lung adenocarcinoma tumor tissues, indicating that the Gas6/Axl signaling pathway was activated in lung adenocarcinoma tumor tissues (25). This also provided a theoretical basis for the present study to investigate the use the Gas6/Axl signaling pathway as a target for the treatment of lung adenocarcinoma. At present, the treatment of advanced lung adenocarcinoma mainly includes chemoradiotherapy, but it can only serve a role in delaying the course of the disease (26). Therefore, the Gas6/Axl signaling pathway may be the focus of future clinical research as a possible therapeutic target for lung adenocarcinoma.

However, there are also certain limitations to the present study. EGFR, echinoderm microtubule-associated protein-like 4, anaplastic lymphoma kinase and VEGF have been indicated to be the most important driver genes of lung adenocarcinoma. However, the specific targets of the Gas6/Axl signaling pathway in lung adenocarcinoma and whether they are associated with the aforementioned driver genes are unknown. Therefore, the specific targets of the Gas6/Axl signaling pathway in lung adenocarcinoma will be explored in subsequent studies to provide a strong theoretical basis for the clinical treatment of lung adenocarcinoma.

In summary, the Gas6/Axl signaling pathway was revealed to be highly expressed in lung adenocarcinoma tissues and cells, and inhibition of the Gas6/Axl signaling pathway attenuated the proliferation and invasion of lung adenocarcinoma cells. The present study provided a potential molecular target for future tumor-targeted therapy and indicated that TP-0903 may be an effective drug to treat lung adenocarcinoma. In addition, the current study preliminarily demonstrated the role of the Gas6/Axl signaling pathway in the development of lung cancer. However, its deeper function and potential molecular mechanisms remain unclear and require further investigation. In conclusion, the Gas6/Axl signaling pathway was indicated to promote the proliferation, migration and invasion and reduce the apoptosis of lung adenocarcinoma cells, thereby serving an important role in the progression of lung adenocarcinoma.

\section{Acknowledgements}

Not applicable.

\section{Funding}

No funding was received.

\section{Availability of data and materials}

All data generated or analyzed during this study are included in this published article.

\section{Authors' contributions}

DW, LB, JR, NX, LZ and XL were responsible for the study design, resources, data curation and analysis. DW and LB were responsible for the manuscript preparation. NX, LZ and XL reviewed and edited the manuscript. DW and XL confirm the authenticity of all the raw data. All authors read and approved the final manuscript.

\section{Ethics approval and consent to participate}

The present study was approved by the Ethics Committee of the Affiliated Hospital of Hebei University (Baoding, China) and written informed consent was provided by all patients.

\section{Patient consent for publication}

Not applicable.

\section{Competing interests}

The authors declare that they have no competing interests.

\section{References}

1. Howlader N, Forjaz G, Mooradian MJ, Meza R, Kong CY, Cronin KA, Mariotto AB, Lowy DR and Feuer EJ: The effect of advances in lung-cancer treatment on population mortality. N Engl J Med 383: 640-649, 2020.

2. Schwartz AG and Cote ML: Epidemiology of lung cancer. Adv Exp Med Biol 893: 21-41, 2016.

3. Yang F, Li H and Wang J: The precision surgical treatment strategies of lung adenocarcinoma under the guidance of the WHO new classification. Zhonghua Wai Ke Za Zhi 55: 49-53, 2017 (In Chinese).

4. Morales-Oyarvide V and Mino-Kenudson M: Tumor islands and spread through air spaces: Distinct patterns of invasion in lung adenocarcinoma. Pathol Int 66: 1-7, 2016.

5. Kometani T, Sugio K, Osoegawa A, Seto T and Ichinose Y: Clinicopathological features of younger (aged $\leq 50$ years) lung adenocarcinoma patients harboring the EML4-ALK fusion gene. Thoracic Cancer 9: 563-570, 2018.

6. Senan S, Paul MA and Lagerwaard FJ: Treatment of early-stage lung cancer detected by screening: Surgery or stereotactic ablative radiotherapy? Lancet Oncol 14: e270-e274, 2013.

7. Jiang $\mathrm{N}$ and $\mathrm{Xu} \mathrm{X}$ : Exploring the survival prognosis of lung adenocarcinoma based on the cancer genome atlas database using artificial neural network. Medicine (Baltimore) 98: e15642, 2019.

8. Chang CH, Lee CH and Wang JY: Gefitinib or erlotinib for previously treated lung adenocarcinoma: Which is superior? J Clin Oncol 35: 1374-1375, 2017. 
9. Gustafsson A, Fritz HKM and Dahlbäck B: Gas6-Axl signaling in presence of Sunitinib is enhanced, diversified and sustained in renal tumor cells, resulting in tumor-progressive advantages. Exp Cell Res 355: 47-56, 2017.

10. Antony J, Tan TZ, Kelly Z, Low J, Choolani M, Recchi C, Gabra H, Thiery JP and Huang RYJ: The GAS6-AXL signaling network is a mesenchymal (Mes) molecular subtype-specific therapeutic target for ovarian cancer. Sci Signal 9: ra97, 2016.

11. Jin Y, Nie D, Li J, Du X, Lu Y, Li Y, Liu C, Zhou J and Pan J Gas6/AXL signaling regulates self-renewal of chronic myelogenous leukemia stem cells by stabilizing beta-catenin. Clin Cancer Res 23: 2842-2855, 2017.

12. Sawabu T, Seno H, Kawashima T, Fukuda A, Uenoyama Y, Kawada M, Kanda N, Sekikawa A, Fukui H, Yanagita M, et al: Growth arrest-specific gene 6 and Axl signaling enhances gastric cancer cell survival via Akt pathway. Mol Carcinog 46: 155-164, 2007.

13. Lee HJ, Jeng YM, Chen YL, Chung L and Yuan RH: Gas6/Axl pathway promotes tumor invasion through the transcriptional activation of slug in hepatocellular carcinoma. Carcinogenesis 35 769-775, 2014

14. Sinha S, Boysen J, Nelson M, Secreto C, Warner SL, Bearss DJ, Lesnick C, Shanafelt TD, Kay NE and Ghosh AK: Targeted Axl inhibition primes chronic lymphocytic leukemia B cells to apoptosis and shows synergistic/additive effects in combination with BTK inhibitors. Clin Cancer Res 21: 2115-2126, 2015.

15. Morizono K, Xie Y, Olafsen T, Lee B, Dasgupta A, Wu AM and Chen IS: The soluble serum protein Gas6 bridges virion envelope phosphatidylserine to the TAM receptor tyrosine kinase Axl to mediate viral entry. Cell Host Microbe 9: 286-298, 2011.

16. Livak KJ and Schmittgen TD: Analysis of relative gene expression data using real-time quantitative PCR and the 2(-Delta Delta C(T)) method. Methods 25: 402-408, 2001.

17. Testa U, Castelli G and Pelosi E. Lung cancers: Molecular characterization, clonal heterogeneity and evolution, and cancer stem cells. Cancers (Basel) 10: 248, 2018.

18. Kariolis MS, Miao YR, Diep A, Nash SE, Olcina MM Jiang D, Jones DS II, Kapur S, Mathews II, Koong AC, et al: Inhibition of the GAS6/AXL pathway augments the efficacy of chemotherapies. J Clin Invest 127: 183-198, 2017.
19. Wang C, Jin H, Wang N, Fan S, Wang Y, Zhang Y, Wei L, Tao X, $\mathrm{Gu}$ D, Zhao F, et al: Gas6/Axl axis contributes to chemoresistance and metastasis in breast cancer through Akt/GSK-3 $\beta / \beta$-catenin signaling. Theranostics 6: 1205-1219, 2016

20. Zhao CC, Chen J, Niu RF, Liu Y and Zhang CG: Increased resistin suggests poor prognosis and promotes development of lung adenocarcinoma. Oncol Rep 40: 3392-33404, 2018.

21. Faget J, Contat C, Zangger N, Peters S and Meylan E: RANKL Signaling sustains primary tumor growth in genetically engineered mouse models of lung adenocarcinoma. J Thorac Oncol 13: 387-398, 2018.

22. Han J, Tian R, Yong B, Luo C, Tan P, Shen J and Peng T: Gas6/Axl mediates tumor cell apoptosis, migration and invasion and predicts the clinical outcome of osteosarcoma patients. Biochem Biophys Res Commun 435: 493-500, 2013.

23. Rankin EB, Fuh KC, Taylor TE, Krieg AJ, Musser M, Yuan J, Wei K, Kuo CJ, Longacre TA and Giaccia AJ: AXL is an essential factor and therapeutic target for metastatic ovarian cancer. Cancer Res 70: 7570-7579, 2010.

24. Sainaghi PP, Castello L, Bergamasco L, Galletti M, Bellosta P and Avanzi GC: Gas6 induces proliferation in prostate carcinoma cell lines expressing the Axl receptor. J Cell Physiol 204: 36-44, 2005.

25. Seike M, Kim CH, Zou F, Noro R, Chiba M, Ishikawa A, Kunugi S, Kubota K and Gemma A: AXL and GAS6 co-expression in lung adenocarcinoma as a prognostic classifier. Oncol Rep 37: 3261-3269, 2017.

26. Scafoglio CR, Villegas B, Abdelhady G, Bailey ST, Liu J, Shirali AS, Wallace WD, Magyar CE, Grogan TR, Elashoff D, et al: Sodium-glucose transporter 2 is a diagnostic and therapeutic target for early-stage lung adenocarcinoma. Sci Transl Med 10: eaat5933, 2018. 